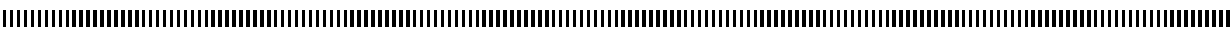

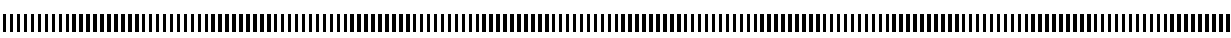
| | | |

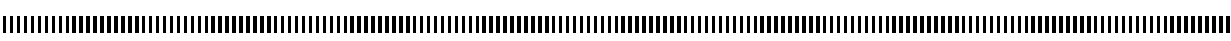

\title{
On a Radially Symmetrical Green's Function
}

\author{
Ould Ahmed Izid Bih Isselkou \\ Université de Nouakchott, B.P. 798 Mauritanie, \\ isselkou@univ-nkc.mr
}

|

ABSTRACT. It is quite usual to transform elliptic PDE problems of second order into fixed point integral problems, via the Green's function. But it is not easy, in general, to handle integrals involved in such a formulation. When it comes to the Laplacian operator on balls of $\Re^{n}$, we give here a radially symmetrical Green's function which, under some nonlinearity assumptions, makes the Green's Integral representation formula easier to use; we give three examples of application.

RÉSUMÉ. II est courant de transformer un problème, donné sous forme d'EDP elliptique de second ordre, en un problème intégral de point fixe, et ce en utilisant la fonction de Green. En général, les intégrales intervenant dans une telle formulation, sont de maniement difficile. Lorsqu'il s'agit de l'opérateur du Laplacien sur des boules de $\Re^{n}$, nous montrons l'existence d'une fonction de Green à symétrie radiale; elle permet, moyennant des hypothèses adéquates sur la non linéarité, de faciliter l'usage de la Formule de représentation de Green; nous donnons trois exemples d'application.

KEYWORDS : Green's function, radially symmetrical, representation formula.

MOTS-CLÉS : Fonction de Green, symétrie radiale, Formule de réprésentation.

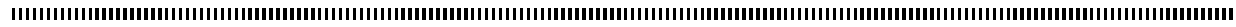




\section{Introduction}

Let us put $B_{r}=\left\{x \in \Re^{n},\|x\|<r\right\}$ and

$$
\Gamma(r)=\left\{\begin{array}{l}
\frac{1}{n w_{n}(2-n)} r^{2-n}, \text { if } n \geq 3, \\
\frac{1}{2 \pi} \log (r), \text { if } n=2 \\
\frac{1}{2} r, \text { if } n=1
\end{array}\right.
$$

where $w_{n}$ is the Lebesgue measure of the unit ball $B_{1}$.

The classical Green's function(cf. [5]) for the Laplacian, on $B_{1}$, is

$G(x, y)=\Gamma(\|x-y\|)-\Gamma\left(\|\| y\left\|x-\frac{y}{\|y\|}\right\|\right)$, if $y \neq O$

$G(x, O)=\Gamma(\|x\|)-\Gamma(1)$.

Let us put

$$
I_{1}(x, y)=G(x, O) \chi_{\{\|y\|<\|x\|\}}(y)+G(O, y) \chi_{\{\|y\|>\|x\|\}}(y),
$$

where the function $\chi$ is the characteristic one. We get

$$
\Delta_{y} I_{1}(x, .)=\delta_{x}, \text { on } \mathcal{D}_{\text {rad }}\left(B_{1}\right),
$$

where $\mathcal{D}_{\text {rad }}\left(B_{1}\right)$ are radially symmetrical test functions and $\delta_{x}$ is the Dirac's distribution at the point $x$.

As examples of application, we deal with the Helmholtz's problem, Talenti's formula and the Lane-Emden function.

\section{The radially symmetrical Green's function}

Definition 2.1 Let $\mathcal{D}\left(B_{1}\right)$ be the set of functions which are $\mathcal{C}^{\infty}$, with compact supports in $B_{1}$ and $\mathcal{D}_{\text {rad }}\left(B_{1}\right)$, the set of functions of $\mathcal{D}\left(B_{1}\right)$, which are radially symmetrical, with respect to the origin.

Remark 2.2 $\forall x \in B_{1}, I_{1}(x,.) \in L^{1}\left(B_{1}\right) \subset \mathcal{D}^{\prime}\left(B_{1}\right)$, where $\mathcal{D}^{\prime}\left(B_{1}\right)$ is the Schwartz distributions set over $B_{1}$.

For every $\varphi \in \mathcal{D}\left(B_{1}\right)$, one defines

$$
M(\varphi)(x)=\frac{1}{\left|\partial B_{\|x\|}\right|} \int_{\partial B_{\|x\|}} \varphi(x) d S(x), M(\varphi)(O)=\varphi(O),
$$

$M(\varphi)$ is the mean of $\varphi$, over the sphere $\partial B_{\|x\|}=\left\{y \in B_{1},\|y\|=\|x\|\right\}$.

Proposition $2.3 \forall x \in B_{1}, \forall \varphi \in \mathcal{D}\left(B_{1}\right), \forall \phi \in \mathcal{D}_{\text {rad }}\left(B_{1}\right)$,

$$
<\Delta_{y} I_{1}(x, .), \varphi>=M(\varphi)(x),<\Delta_{y} I_{1}(x, .), \phi>=<\delta_{x}, \phi>,
$$

where $\delta_{x}$ is the Dirac distribution at the point $x$. 
Proof.

If $n \geq 3, \forall x \in B_{1}$, we have

$$
\begin{gathered}
\left\langle\Delta_{y} I(x, .), \varphi>=\right. \\
c_{n}\left\{\left(\|x\|^{2-n}-1\right) \int_{B_{\|x\|}} \Delta \varphi(y) d y+\int_{B_{1}-\bar{B}_{\|x\|}}\left(\|y\|^{2-n}-1\right) \Delta \varphi(y) d y\right\} .
\end{gathered}
$$

If $x=O$, we have only the second integral, $c_{n} \int_{B_{1}}\left(\|y\|^{2-n}-1\right) \Delta \varphi(y) d y$. As $\Delta_{y}\left(c_{n}\left(\|y\|^{2-n}-1\right)\right)=\delta_{O}$, we get

$$
\begin{gathered}
c_{n} \int_{B_{1}}\left(\|y\|^{2-n}-1\right) \Delta \varphi(y) d y=<c_{n} \Delta\left(\|y\|^{2-n}-1\right), \varphi>=c_{n}<\Delta\left(\|y\|^{2-n}-1\right), \varphi> \\
=<\delta_{O}, \varphi>=\varphi(O) .
\end{gathered}
$$

Let us put $\psi(y)=\|y\|^{2-n}-1 ; \Delta \psi=0$, in $B_{1}-\bar{B}_{\|x\|}$. Using the Divergence Theorem and the Green's Identity, we get

$$
\begin{gathered}
\left\langle\Delta_{y} I_{1}(x, .), \varphi>=\right. \\
c_{n}\left\{\left(\|x\|^{2-n}-1\right) \int_{\partial B_{\|x\|}} \frac{\partial \varphi(y)}{\partial \nu} d s+\int_{\partial\left(B_{1}-\bar{B}_{\|x\|}\right)}\left(\psi(y) \frac{\partial \varphi}{\partial \nu}(y)-\varphi(y) \frac{\partial \psi}{\partial \nu}(y)\right) d s\right\},
\end{gathered}
$$

where $\nu$ is the outer normal. Using the fact that $\psi(y)=\varphi(y)=0$, if $y \in \partial B_{1}$, we get

$$
\begin{gathered}
<\Delta_{y} I_{1}(x, .), \varphi>=c_{n}\left\{\left(\|x\|^{2-n}-1\right) \int_{\partial B_{\|x\|}} \frac{\partial \varphi(y)}{\partial \nu} d s\right. \\
-\int_{\partial B_{\|x\|}}\left(\psi(y) \frac{\partial \varphi}{\partial \nu}(y)-\varphi(y) \frac{\partial \psi}{\partial \nu}(y)\right\} d s
\end{gathered}
$$

As $\forall y \in \partial B_{\|x\|}, \psi(y)=\|x\|^{2-n}-1$ and $\frac{\partial \psi}{\partial \nu}(y)=(2-n)\|x\|^{1-n}$, we obtain

$$
\begin{gathered}
<\Delta_{y} I_{1}(x, .), \varphi>=c_{n} \int_{\partial B_{\|x\|}} \varphi(y)(2-n)\|x\|^{1-n} d s=c_{n}(2-n)\|x\|^{1-n} \int_{\partial B_{\|x\|}} \varphi(y) d s \\
=M(\varphi)(x) .
\end{gathered}
$$

If $\phi$ is radially symmetrical, we infer that

$$
<\Delta_{y} I(x, .), \phi>=\phi(\|x\|) c_{n}\|x\|^{1-n}(2-n)\left|\partial B_{\|x\|}\right|=\phi(\|x\|) .
$$

If $n=2$, then, as in the case $n \geq 3$, we use the Divergence Theorem, the Green's Identity and set $\psi(y)=\log \|y\|$. We get

$$
\begin{aligned}
<\Delta_{y} I_{1}(x, .), \varphi>=\frac{1}{2 \pi}\left(\log \|x\| \int_{B_{\|x\|}} \Delta \varphi(y) d y+\int_{B_{1}-\overline{B_{\|x\|}}} \psi(y) \Delta \varphi(y) d y\right) \\
=\frac{1}{2 \pi}\left(\log \|x\| \int_{\partial B_{\|x\|}} \frac{\partial \varphi}{\partial \nu}(y) d s-\int_{\partial B_{\|x\|}} \psi(y) \frac{\partial \varphi}{\partial \nu}(y) d s+\int_{\partial B_{\|x\|}} \varphi(y) \frac{\partial \psi}{\partial \nu}(y) d s\right)
\end{aligned}
$$




$$
=\frac{1}{2 \pi} \frac{1}{\|x\|} \int_{\partial B_{\|x\|}} \varphi(y) d s=M(\varphi)(x) .
$$

If $\phi \in D_{\text {rad }}\left(B_{1}\right)$, we obtain

$$
<\Delta_{y} I_{1}(x, .), \phi>=M(\phi)(x)=\frac{1}{2 \pi} \frac{1}{\|x\|}\left|\partial B_{\|x\|}\right| \phi(\|x\|)=\phi(\|x\|) .
$$

If $n=1$, let $\varphi$ be an even (radially symmetrical) function, which belongs to $\mathcal{D}(]-1,1[)$, then

$$
\begin{aligned}
<\Delta_{y} I_{1}(t, .), \varphi> & =<I_{1}(t, .), \varphi^{\prime \prime}> \\
& =\frac{1}{2}\left((|t|-1) \int_{-|t|}^{|t|} \varphi^{\prime \prime}(s) d s+\int_{|s|>|t|}(|s|-1) \varphi^{\prime \prime}(s) d s\right) \\
& =\frac{1}{2}\left((|t|-1) \int_{-|t|}^{|t|} \varphi^{\prime \prime}(s) d s-\int_{-1}^{-|t|}(s+1) \varphi^{\prime \prime}(s) d s\right. \\
& \left.+\int_{|t|}^{1}(s-1) \varphi^{\prime \prime}(s) d s\right) \\
& \left.=\frac{1}{2}\left((|t|-1) \varphi^{\prime}(s)\right]_{-|t|}^{|t|}-(s+1) \varphi^{\prime}(s)\right]_{-1}^{-|t|} \\
& \left.\left.+(s-1) \varphi^{\prime}(s)\right]_{|t|}^{1}+\int_{-1}^{-|t|} \varphi^{\prime}(s) d s-\int_{|t|}^{1} \varphi^{\prime}(s) d s\right)
\end{aligned}
$$

As $\varphi(-1)=\varphi(1)=0$, we get

We then get

$$
\begin{gathered}
<\Delta_{y} I_{1}(t, .), \varphi>=\frac{1}{2}\left((|t|-1)\left(\varphi^{\prime}(|t|)-\varphi^{\prime}(-|t|)\right)-(-|t|+1) \varphi^{\prime}(-|t|)\right) \\
\left.+\frac{1}{2}\left(-(|t|-1) \varphi^{\prime}(|t|)+\varphi(-|t|)+\varphi(|t|)\right)\right) \\
=\frac{1}{2}(\varphi(-|t|)+\varphi(|t|))=M(\varphi)(t) .
\end{gathered}
$$

$$
<\Delta_{y} I_{1}(t, .), \phi>=M(\phi)(t)=\phi(|t|) .
$$

If one considers a ball $B_{r}$ of radius $r>0$, instead of $B_{1}$, and defines

$$
\begin{gathered}
I_{r}(x, y)=c_{n}\left(\left(\|x\|^{2-n}-r^{2-n}\right) \chi_{B_{\|x\|}}(y)+\left(\|y\|^{2-n}-r^{2-n}\right) \chi_{B_{r}-\bar{B}_{\|x\|}}(y)\right), \text { for } n \geq 3, \\
I_{r}(x, y)=\frac{1}{2 \pi}\left((\log \|x\|-\log r) \chi_{B_{\|x\|}}(y)+(\log \|y\|-\log r) \chi_{\left.B_{r}-\bar{B}_{\|x\|}(y)\right), \text { for } n=2,},\right. \\
I_{r}(x, y)=\frac{1}{2}\left((|x|-r) \chi_{\{|y|<|x|\}}(y)+(|y|-r) \chi_{\{|x|<|y|\}}(y)\right), \text { for } n=1,
\end{gathered}
$$

we obtain,

Proposition $2.4 \forall \varphi \in D\left(B_{r}\right)$ and $\forall \phi \in D_{\operatorname{rad}}\left(B_{r}\right)$,

$$
<\Delta_{y} I_{r}(x, .), \varphi>=M(\varphi)(x) \text { and }<\Delta_{y} I_{r}(x, .), \phi>=<\delta_{x}, \phi>.
$$

Proof.

The same proof, as in the unit ball case(cf. Proposition 1).

Remark 2.5 Since $I_{r}(x, y)=I_{r}(\|x\|,\|y\|)$, we have called it a Radially Symmetrical Green's Function. 


\section{Applications}

Let us give three examples of applications.

\subsection{The Helmholtz's problem}

Let us suppose $n \geq 3$ and look for $(u, \lambda>0)$, solution of

$$
(*)\left\{\begin{array}{l}
-\Delta u(x)=\lambda u(x), \text { in } B_{1} \subset \Re^{n}, \\
u=0 \text { on } \partial B_{1},
\end{array}\right.
$$

We put

$$
v(r)=a_{0} \sum_{k=0}^{\infty}(-1)^{k} \frac{1}{2^{k} k ! \Pi_{j=0}^{k-1}(n+2 j)} r^{2 k} .
$$

Proposition 3.1 The problem $(*)$ admits the analytical solutions $u_{\lambda}(r)=v(\sqrt{\lambda} r)$, where $\lambda$ is any positive "zero" of $v$.

Proof.

One can use results from [4], to see that every regular solution of $(*)$ is radially symmetrical, with respect to the origin. Using the polar coordinates, one can rewrite the problem $(*)$ as following

$$
\left\{\begin{array}{l}
-\left(u ”(r)+\frac{2}{r} u^{\prime}(r)\right)=\lambda u(r), r \text { in }[0,1[, \lambda>0 \\
u(1)=0 .
\end{array}\right.
$$

Using Frobenius's theorem, one infers the previous problem admits a analytical solution $v$ near the origin. Let us suppose the convergence radius of $v$ greater than 1 , using $I_{1}$ instead of the classical Green's function, we get that any solution of (*) can be written as follows

$$
\begin{gathered}
u(r)=-\lambda \int_{B_{1}} I_{1}(x, y) u(y) d y \\
=-\lambda c_{n}\left(\int_{\|x\|>\|y\|}\left(\|x\|^{2-n}-1\right) u(\|y\|) d y+\int_{\|x\|<\|y\|}\left(\|y\|^{2-n}-1\right) u(\|y\|) d y\right) .
\end{gathered}
$$

Using polar coordinates, we get

$$
u(r)=-\frac{\lambda}{2-n}\left(\left(r^{2-n}-1\right) \int_{0}^{\|x\|} s^{n-1} u(s) d s+\int_{\|x\|}^{1}\left(s^{2-n}-1\right) s^{n-1} u(s) d s\right) .
$$

Replacing $u(s)$ by the unknown series, $\sum_{k=0}^{\infty} a_{k} r^{k}$, interchanging the signs $\int$ and $\Sigma$ and performing standard computations, one obtains

$$
a_{2 k+1}=0 \text { and } a_{2 k}=a_{0}(-1)^{k} \lambda^{k} \frac{1}{2^{k} k ! \Pi_{j=0}^{k-1}(n+2 j)}, \forall k>1 .
$$

So we get $u(r)=v(\sqrt{\lambda} r)$ and this ends the proof. 
Remark 3.2 If $n=3$, as $2^{k} k ! \Pi_{j=0}^{k-1}(n+2 j)=(2 k+1)$ !, we get the wellknown result:

$$
u_{\lambda}(r)=a_{0} \frac{\sin (\sqrt{\lambda} r)}{\sqrt{\lambda} r} \text { and } \lambda=j^{2} \pi^{2}, j>1 .
$$

If $n=4$, as $2^{k} k ! \Pi_{j=0}^{k-1}(n+2 j)=2^{2 k} k !(k+1) !$, another wellknown result,

$$
u_{\lambda}(r)=\frac{a_{0}}{\sqrt{\lambda} r} J_{1}(\sqrt{\lambda} r),
$$

where $\lambda$ is any positive zero of Bessel's first kind function $J_{1}$. If we use the "fzero" subroutine in Matlab, the first zero of $J_{1}$ is about 3.8317.

\subsection{Talenti's formula}

The Talenti's formula gives an isoperimetric inequality for solutions of rearranged linear elliptic problems (cf. [13]).

Let us consider the semilinear elliptic problem

$$
\text { (1) }\left\{\begin{array}{l}
\Delta u(x)+g(\|x\|, u(x))=0, \text { in } B_{1} \subset \Re^{3}, \\
u=0 \text { on } \partial B_{1},
\end{array}\right.
$$

where $g$, say, is continuous. Let $u$ be a radially symmetrical nonnegative solution(cf.[4]) of (1), we have

\section{Proposition 3.3}

$$
u(x)=\frac{1}{n^{2} w_{n}^{\frac{2}{n}}} \int_{w_{n}\|x\|^{n}}^{w_{n}} t^{\frac{2}{n}-2} \int_{0}^{t} g\left(\left(\frac{r}{w_{n}}\right)^{\frac{1}{n}}, u\left(\left(\frac{r}{w_{n}}\right)^{\frac{1}{n}}\right)\right) d r d t .
$$

Proof.

Replacing the classical Green's function $G$ (for $\Delta$, on $B_{1}$ ), by the new one $I_{1}$, and using again the Green's Representation Formula, we get

$$
\begin{gathered}
u(x)=-\int_{B_{1}} I_{1}(x, y) g(y, u(y)) d y \\
=c_{n}\left\{\left(1-\|x\|^{2-n}\right) \int_{B_{\|x\|}} g\left(y, u(y) d y+\int_{B_{1}-\overline{B_{\|x\|}}}\left(1-\|y\|^{2-n}\right) g(y, u(y)) d t\right\}\right. \\
=\frac{1}{2-n}\left\{\left(1-\|x\|^{2-n}\right) \int_{0}^{\|x\|} r^{n-1} g\left(r, u(r) d r+\int_{\|x\|}^{1} r^{n-1}\left(1-r^{2-n}\right) g(r, u(r)) d r\right\}\right. \\
=\int_{\mid x \|}^{1} s^{1-n} d s \int_{0}^{\|x\|} r^{n-1} g\left(r, u(r) d r+\int_{\|x\|}^{1} r^{n-1} \int_{r}^{1} s^{1-n} d s g(r, u(r)) d r .\right.
\end{gathered}
$$

Interchanging the order of integration( by Fubini's Theorem), we get

$$
u(x)=\int_{\|x\|}^{1} s^{1-n} \int_{0}^{s} r^{n-1} g(r, u(r)) d r d s .
$$


If we put $w_{n} r^{n}=t$ (and use $r$ again), we get

$$
u(x)=\frac{1}{n w_{n}} \int_{\|x\|}^{1} s^{1-n} \int_{0}^{w_{n} s^{n}} g\left(\left(\frac{r}{w_{n}}\right)^{\frac{1}{n}}, u\left(\left(\frac{r}{w_{n}}\right)^{\frac{1}{n}}\right)\right) d r d s .
$$

Putting $t=w_{n} s^{n}$ (and using $s$ again), we get

$$
u(x)=\frac{1}{n^{2} w_{n}^{\frac{2}{n}}} \int_{w_{n}\|x\|^{n}}^{w_{n}} s^{\frac{2}{n}-2} \int_{0}^{s} g\left(\left(\frac{r}{w_{n}}\right)^{\frac{1}{n}}, u\left(\left(\frac{r}{w_{n}}\right)^{\frac{1}{n}}\right)\right) d r d s .
$$

Let us now suppose $g(r, u)$ is a nonnegative function, decreasing with respect to the first variable and increasing with respect to the second one or $g(r, u)=f(r)$, where $f$ is a nonnegative decreasing function. We also suppose $g(r, 0) \neq 0$, then we get Talenti's formula

Corollary 3.4 For every nonnegative solution $u$ of (1), we have

$$
u(x)=\frac{1}{n^{2} w_{n}^{\frac{2}{n}}} \int_{|u>u(x)|}^{\left|B_{1}\right|} t^{\frac{2}{n}-2} F(t) d t
$$

where $F(t)=\int_{0}^{t} h_{*}(s) d s, h_{*}$ is the decreasing rearrangement of the function $h(r)=g(r, u(r))$ and $w_{n}=\left|B_{1}\right|$.

Proof.

Using the Maximum Principle and [4], we see that any nonnegative solution $u$ of (1) is a radially decreasing function and $h(r)=g(r, u(r))$ is also a decreasing function. The standard properties of the rearrangement(cf [13]) give us

$$
\int_{0}^{r} h\left(\left(\frac{s}{w_{n}}\right)^{\frac{1}{n}}\right) d r=\int_{0}^{r} h_{*}(s) d s .
$$

As $u$ is radially decreasing, we get $w_{n}\|x\|^{n}=|u>u(x)|$. So one can use the previous Proposition to end the proof.

\subsection{The Lane-Emden function}

Let us consider the semilinear problem,

$$
\left(P_{\lambda}\right)\left\{\begin{array}{l}
\Delta u+\lambda(1+u)^{2}=0 \text { in } B_{1} \subset \Re^{3} \\
u \geq 0, \text { in } B_{1} \\
u=0 \text { on } \partial B_{1} .
\end{array}\right.
$$

This Problem is a particular case of a class of mathematical models (cf.[3]). It is known (cf. [11],[3], [1],[8], [10]) that there exists a critical eigenvalue $\lambda^{*}(2)$, such that $\left(P_{\lambda}\right)$ admits two solutions if $0<\lambda<\lambda^{*}(2)$, one solution if $\lambda=\lambda^{*}(2)$ and no solution if $\lambda>\lambda^{*}(2)$. The minimal solution is analytical(cf. [12],[10], [9]). Let $u$ be a solution of $\left(P_{\lambda}\right)$, then $u$ is a radially symmetrical decreasing function (cf. [4]).

Let $\varphi$ be the Lane-Emden function (cf.[2],[6],[7] and [14]), solution of

$$
(E)\left\{\begin{array}{l}
v^{\prime \prime}(r)+2 v^{\prime}(r)+r v^{2}(r)=0 \\
v(0)=1, v^{\prime}(0)=0
\end{array}\right.
$$


Proposition 3.5 Let us suppose $0<\lambda \leq \lambda^{*}(2)$, if $u$ is the minimal solution of $\left(P_{\lambda}\right)$, then

$$
u(r)=\frac{c}{\lambda} \varphi(r \sqrt{c})-1
$$

where $c$ is such that $\lambda=c \varphi(\sqrt{c})$.

Proof.

Let $u(r)=\sum_{i=0}^{\infty} a_{i} r^{i}$, be the minimal solution (near 0 ) of $\left(P_{\lambda}\right)$, then using $I_{1}$ instead of $G$, we get

$$
\Sigma_{i=0}^{\infty} a_{i} r^{i}=\lambda\left(\left(r^{-1}-1\right) \int_{0}^{r} t^{2} \Sigma_{j=0}^{\infty} c_{j} t^{j} d t+\int_{r}^{1} t^{2}\left(t^{-1}-1\right) \Sigma_{j=0}^{\infty} c_{j} t^{j} d t\right)
$$

where $\Sigma_{j=0}^{\infty} c_{j} r^{j}=(1+u(r))^{2}$. We get

$$
\begin{gathered}
\sum_{i=0}^{\infty} a_{i} r^{i}=\lambda\left(\left(r^{-1}-1\right) \int_{0}^{r} t^{2} \sum_{j=0}^{\infty} c_{j} t^{j} d t+\int_{r}^{1}\left(t-t^{2}\right) \Sigma_{j=0}^{\infty} c_{j} t^{j} d t\right) \\
=\lambda\left(\Sigma_{j=0}^{\infty} c_{j} \frac{r^{2+j}}{3+j}-\Sigma_{j=0}^{\infty} c_{j} \frac{r^{3+j}}{3+j}+\Sigma_{j=0}^{\infty} \frac{c_{j}}{j+2}-\Sigma_{j=0}^{\infty} \frac{c_{j}}{3+j}\right. \\
\left.-\Sigma_{j=0}^{\infty} c_{j} \frac{r^{2+j}}{2+j}+\Sigma_{j=0}^{\infty} c_{j} \frac{r^{3+j}}{3+j}\right) \\
=\lambda\left(\Sigma_{j=2}^{\infty} c_{j-2} \frac{r^{j}}{1+j}+\Sigma_{j=0}^{\infty} \frac{c_{j}}{j+2}-\Sigma_{j=0}^{\infty} \frac{c_{j}}{3+j}-\Sigma_{j=2}^{\infty} c_{j-2} \frac{r^{j}}{j}\right) \\
=\lambda\left(\sum_{j=2}^{\infty} c_{j-2} r^{j}\left(\frac{1}{1+j}-\frac{1}{j}\right)+\Sigma_{j=0}^{\infty} c_{j}\left(\frac{1}{2+j}-\frac{1}{j+3}\right)\right), \\
c_{j}=\Sigma_{k=0}^{j} a_{k}^{\prime} a_{j-k}^{\prime}, \text { with } a_{i}^{\prime}=a_{i}, \forall i \neq 0 \text { and } a_{0}^{\prime}=1+a_{0} .
\end{gathered}
$$

We infer that,

$$
a_{0}=\Sigma_{j=0}^{\infty} c_{j}\left(\frac{1}{2+j}-\frac{1}{j+3}\right), a_{1}=0 \text { and } a_{j}=\lambda\left(\frac{1}{1+j}-\frac{1}{j}\right) c_{j-2}, \text { if } j>2 .
$$

Let us put,

$$
d_{0}=1, d_{2 i}=\left(\frac{1}{2 i+1}-\frac{1}{2 i}\right) \Sigma_{k=0}^{i-1} d_{2 k} d_{2 i-2-2 k}, \text { and } d_{2 i+1}=0, \forall i \geq 1 .
$$

By induction, we see that

$$
a_{2 i}=d_{2 i} \lambda^{i}\left(1+a_{0}\right)^{i+1} \text { and } a_{2 i+1}=0, \forall i \geq 1 .
$$

Let us put $\varphi(r)=\Sigma_{i=0}^{\infty} a_{2 i} r^{2 i}$, one can use (1) to infer that

$$
\varphi^{2}(r)=-\frac{1}{r}(r \varphi(r)) "
$$

As $n=3$, we have

$$
\frac{1}{r}(r \varphi(r)) "=\Delta \varphi(r) .
$$


So we infer that $\varphi$ is the Lane-Emden function, for $\varphi(0)=d_{0}=1, \varphi^{\prime}(0)=d_{1}=0$. We also get

$$
\begin{gathered}
u(r)=\sum_{i=0}^{\infty} a_{i} r^{i}=a_{0}+\sum_{i=1}^{\infty} d_{2 i} \lambda^{i}\left(1+a_{0}\right)^{i+1} r^{2 i} \\
=a_{0}+1+\sum_{i=1}^{\infty} d_{2 i} \lambda^{i}\left(1+a_{0}\right)^{i+1} r^{2 i}-1 \\
=\frac{1}{\lambda} \lambda\left(1+a_{0}\right) \sum_{i=0}^{\infty} d_{2 i} \lambda^{i}\left(1+a_{0}\right)^{i} r^{2 i}-1=\frac{1}{\lambda} c \varphi(r \sqrt{c})-1,
\end{gathered}
$$

where $c=\lambda\left(1+a_{0}\right)$. We have $u(1)=\frac{c}{\lambda} \varphi(\sqrt{c})-1=0$ or $\lambda=c \varphi(\sqrt{c})$.

\section{Proposition 3.6 The power series}

$$
\sum_{i=0}^{\infty} d_{2 i} r^{2 i}
$$

is alternating and has a radius of convergence $r_{0} \geq 1$.

\section{Proof.}

$d_{0}=1$ and $d_{2}=-\frac{1}{6}$, we will infer that $d_{2 i}=(-1)^{i}\left|d_{2 i}\right|$. Let us suppose the previous relation is true for every $i \in[0 \mathrm{n}]$, then

$$
d_{2(n+1)}=-\frac{1}{(2(n+1)+1)(2 n)} \sum_{i=0}^{n} d_{2 i} d_{2(n+1-i)} .
$$

Using the recurrence hypothesis, we get

$$
d_{2(n+1)}=-(-1)^{n} \frac{1}{(2(n+1)+1)(2 n)} \sum_{i=0}^{n}\left|d_{2 i}\right|\left|d_{2(n-i)}\right|,
$$

which gives the conclusion.

Let us now show that

$$
\lim _{n \rightarrow \infty} d_{2 n}=0 \text { and }\left|d_{2(n+1)}\right|<\left|d_{2 n}\right| .
$$

It is immediate to see(by induction) that $\left|d_{2 n}\right| \leq 1$. So we get

$$
\begin{aligned}
\left|d_{2(n+1)}\right| & =\frac{1}{(2(n+1)+1)(2 n+2)} \sum_{i=0}^{n}\left|d_{2 i}\right|\left|d_{2(n+1-i)}\right| \\
& \leq \frac{1}{(2(n+1)+1)(2 n+2)}(n+1) \\
& \leq \frac{1}{2(2(n+1)+1)} \rightarrow 0, \text { as } n \rightarrow \infty .
\end{aligned}
$$

We have, $\left|d_{2}\right|=\frac{1}{6}<d_{0}=1$, let us suppose that

$$
\begin{gathered}
\left|d_{2(i+1)}\right|<\left|d_{2 i}\right|, \quad \forall 0 \leq i \leq n . \\
\left|d_{2 n}\right|=\frac{1}{(2 n+1)(2 n)} \sum_{i=0}^{n-1}\left|d_{2 i}\right|\left|d_{2(n-1-i)}\right| .
\end{gathered}
$$

Using the recurrence hypothesis $\left(\left|d_{2(n-1-i)}\right| \geq\left|d_{2(n-i)}\right|\right)$, we get

$$
\left|d_{2 n}\right| \geq \frac{1}{(2 n+1)(2 n)} \sum_{i=0}^{n-1}\left|d_{2 i}\right|\left|d_{2(n-i)}\right|
$$




$$
=\frac{1}{(2 n+1)(2 n)}\left((2 n+3)(2(n+1))\left|d_{2(n+1)}\right|-\left|d_{2 n}\right|\right) .
$$

So we infer that

$$
\left|d_{2(n+1)}\right| \leq \frac{4 n^{2}+2 n+1}{4 n^{2}+10 n+6}\left|d_{2 n}\right|<\left|d_{2 n}\right| .
$$

We deduce from the previous inequality that $r_{0} \geq 1$. We have

$$
\frac{1}{r_{0}}=\limsup _{n \rightarrow \infty}\left|d_{2 n}\right|^{\frac{1}{2 n}}=\lim _{n \rightarrow \infty}\left|d_{2 n}\right|^{\frac{1}{2 n}}
$$

Let us consider the problem,

$$
(P)\left\{\begin{array}{l}
\Delta v+v^{2}=0 \text { in } B_{1}, \\
v>0 \text { in } B_{1} \\
v=0 \text { on } \partial B_{1},
\end{array}\right.
$$

Proposition 3.7 Let $u$ be the unique solution of $(P)$, then $\sqrt{u(0)}$ is the first zero of $\varphi$.

Proof.

Let $u$ be the unique solution of $(P)$ and let us define $v(x)=\frac{1}{u(0)} u\left(\frac{x}{\sqrt{u(0)}}\right)$. As the function $u$ is radially symmetrical and analytical on $[0,1]$, so is $v$ on $[0, \sqrt{u(0)}]$. The Maximum Principle implies that,

$v(r)>0$ forall $0 \leq r<\sqrt{u(0)}, v(\sqrt{u(0)})=\frac{1}{u(0)} u(1)=0$. As $u^{\prime}(0)=0$, we get $v^{\prime}(0)=0$. As $v(0)=1$ and $v$ verifies

$$
\Delta v(x)+v^{2}(x)=0,
$$

we get

$$
v(r)=\varphi(r), \forall 0 \leq r \leq \sqrt{u(0)} .
$$

\section{Numerical Computations}

Let us call $\xi_{1}$, the first zero of $\varphi$. From a modelling point of view, the constants $\xi_{1}$ and $r_{0}$ are important, numerical estimations of these constants exist (cf. ([2], p. 95) and ([7], p. 360)).

As

$$
r_{0}=\lim _{n \rightarrow \infty}\left|d_{2 n}\right|^{\frac{-1}{2 n}}
$$

we have computed (using Maple) some values of $\left|d_{2 n}\right|^{\frac{-1}{2 n}}$ and obtained 


\begin{tabular}{l|r}
$n$ & $\left|d_{2 n}\right|^{\frac{-1}{2 n}}$ \\
\hline 200 & 3.908246029 \\
300 & 3.924291150 \\
400 & 3.932914538 \\
500 & 3.938350910 \\
600 & 3.942113057 \\
700 & 3.944881693 \\
800 & 3.947010199 \\
900 & 3.948701028 \\
1000 & 3.950078638 \\
1100 & 3.951224204
\end{tabular}

In order to get an estimation of $\lambda^{*}(2)$, one can use Proposition 5, plot the curve $r \rightarrow r^{2} \varphi(r)$, on the interval $\left[0, \xi_{1}\right]$ and get its maximum.

\section{References}

[1] BREZIS H., NIRENBERG L., " Positive Solutions of Nonlinear Elliptic Equations Involving Critical Sobolev Exponent ”, Comm. Pure Appl. Math., vol. 36 (1983), 437-477.

[2] CHANDRASEKHAR S. , "An Introduction to the Study of Stellar Structure ”, University of Chicago Press, Chicago, 1939.

[3] CRANDALL M.G., RABINOWITZ P.H., "Some Continuation and Variational Methods for Positive Solutions of Nonlinear Elliptic Eigenvalue Problems ", Arch. Rational Mech. Anal., vol. 58, (1975), 207-218.

[4] GIDAS B., NI W.-M., , NI W.-M., " Symmetry and Related Properties via the Maximum Principle ”, Comm. Math. Phys., vol. 68, (1979), 209-243.

[5] GILBARG D., TRUDINGER N.S., “Elliptic Partial Differential Equations of Second Order", Springer Verlag, 1983.

[6] GOENNER H., HAVAS P., "Exact Solutions of the Generalized Lane-Emden Equation ”, $J$. Math. Phys., vol. 41, num. 10, (2000), 729-742.

[7] HOREDT G.P., " Approximate Analytical Solutions of the Lane-Emden Equation in Ndimensional Space”, Astron. Astrophys., vol. 172, (1987), 359-367.

[8] ISSELKOU O.A.-I.-B., "Critical Boundary Constants and Pohozaev Identity", Ann. Fac. Sc. Toulouse, vol. 10, num. 1, (2001), 347-359.

[9] ISSELKOU O.A.-I.-B., “ Critical Eigenvalues for a Nonlinear Problem ”, Nonlinear Differ. Equ. appl., vol. 11, (2004), 225-236.

[10] JOSEPH D.D., LUNDGREN T.S., "Quasilinear Dirichlet Problems Driven by Positive Sources”, Arch. Rational Mech. Anal., vol. 49, (1973), 241-269.

[11] KELLER H.B., "Some Positone Problems Suggested by the Nonlinear Heat Generation", in Bifurcation Theory and Nonlinear Eigenvalues Problems W.A. BENJAMIN, INC., 1969.

[12] MOSSINO J., "Inégalités Isopérimétriques et Applications en Physique", Hermann, Paris, 1984.

[13] SACHDEV P.L., "Nonlinear Ordinary Differential Equations and Their Applications", Pure and Applied Mathematics, vol. 142. 